\title{
Novel Interactive Techniques for Bimanual Manipulation of 3D Objects with Two 3DoF Haptic Interfaces
}

\author{
Anthony Talvas, Maud Marchal, Clément Nicolas, Gabriel Cirio, Mathieu \\ Emily, Anatole Lécuyer \\ IRISA/INRIA Rennes, France \\ \{anthony.talvas, maud.marchal, anatole.lecuyer\}@inria.fr
}

\begin{abstract}
This paper presents a set of novel interactive techniques adapted to two-handed manipulation of objects with dual 3DoF singlepoint haptic devices. We first propose the double bubble for bimanual haptic exploration of virtual environments through hybrid position/rate controls, and a bimanual viewport adaptation method that keeps both proxies on screen in large environments. We also present two bimanual haptic manipulation techniques that facilitate pick-and-place tasks: the joint control, which forces common control modes and control/display ratios for two interfaces grabbing an object, and the magnetic pinch, which simulates a magnet-like attraction between both hands to prevent unwanted drops of that object. An experiment was conducted to assess the efficiency of these techniques for pick-and-place tasks, by comparing the double bubble with viewport adaptation to the clutching technique for extending the workspaces, and by measuring the benefits of the joint control and magnetic pinch.
\end{abstract}

Keywords: Haptic interfaces, Bimanual manipulation, Virtual manipulation, Interaction techniques

\section{Introduction}

In the field of haptics and virtual reality, two-handed interaction with virtual environments (VEs) is a domain that is slowly emerging while bearing very promising applications. Examples of these are surgery training [1], rehabilitation [2], industrial prototyping [3], and 3D graphics [4]. More generally, the use of two hands in haptics allows to realize tasks in a more natural way, as most tasks done in real life are bimanual in a way or another: from simple cases such as using scissors to more complex ones such as playing the guitar.

Numerous haptic devices have been proven to be suitable for bimanual interaction, which can be either single-point interfaces, in which case they will be represented by proxies in the VE, or multi-fingered interfaces, with which it is much easier to mimic the behaviors of an actual hand. Most of these interfaces, however, have small workspaces, which is a strong limitation when a user wants to carry out tasks as simple as pick-and-placing an object in a large VE. Several 
hardware and software solutions were proposed to address this issue, notably the Bubble technique [18] which proved to be suitable for simultaneous grasping of objects and exploration in a VE with a bimanual whole-hand haptic interface [9]. However, while multi-fingered interfaces make it easier to firmly hold an object with two hands through the use of the fingers, it is still currently very difficult to grab and carry an object with proxies controlled by two single-point haptic interfaces, especially in large environments. Notably, a grasped object tends to slip from virtual hands if the contacts between them are not strongly maintained, and current bimanual navigation techniques tend to add to the difficulty of keeping those contacts over time.

In this paper, we introduce novel metaphors and interaction techniques to improve bimanual interaction with dual single-point haptic interfaces. Our major contributions are a double bubble technique with viewport adaptation for bimanual haptic exploration of large VEs, as well as the magnetic pinch and joint control techniques for facilitating the grasping and carrying of virtual objects with two virtual proxies. The paper is structured as follows: section 2 covers the related work on bimanual interaction with large VEs, section 3 details the proposed techniques, section 4 presents the experiment conducted to evaluate the techniques and section 5 discusses the results of the evaluation. Finally, conclusion and perspectives are presented in section 6 .

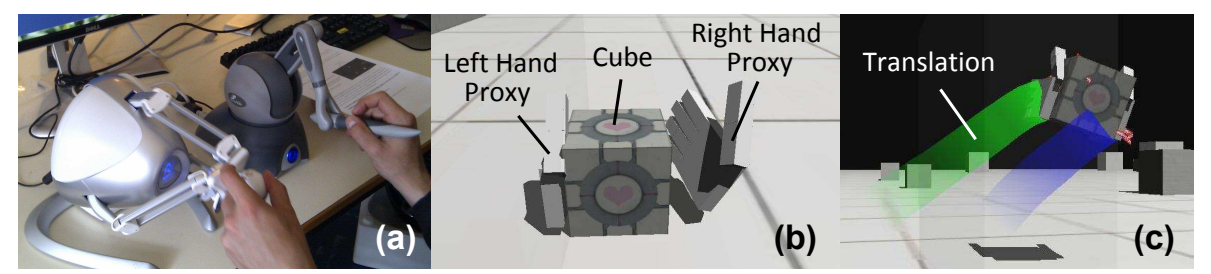

Fig. 1. Example of a bimanual pick-and-place task in a large VE as addressed in this paper. (a) Bimanual haptic setup made of two single-point devices. (b) Grasping a virtual cube with two proxies. (c) Carrying and displacing the cube using our novel interactive techniques.

\section{Related Work}

This section presents the current state of the art in bimanual haptic interaction in large VEs, by first giving an overview of the existing bimanual haptic devices, then exposing the previously proposed hardware and software solutions for extending their workspaces, notably the bubble technique, and finally evoking the grasping of objects with two single-point haptic interfaces.

Several haptic devices allow bimanual interaction with VEs, whether being devices that were specifically designed for such use, or generic devices that were either adapted to this context or used as is. Some are single-point interfaces, such as the SPIDAR G\&G [5], the DLR bimanual haptic interface [3] or the more widespread PHANToM series. Others are multi-fingered interfaces, such as the MasterFinger-2 [6], SPIDAR-8 [7] and Bimanual HIRO [8], which enable 
interaction through 4, 8 and 10 fingertips respectively. Within this category of multi-fingered devices, the Haptic Workstation is a special case, not only providing interaction through the fingertips but also through the palm of both hands [9]. Multi-fingered interfaces are especially suited for grasping virtual objects thanks to their numerous interaction points, although it was shown that single-point devices could also allow grasping with a full control of all degrees of freedom (DoF) of an object through a soft-fingers method [10]. A disadvantage of the aforementioned devices is that their workspaces are limited, and as such are not well suited for working in large VEs, hence bringing the need for techniques that increase the available workspace.

Existing solutions for increasing the workspace of bimanual interfaces can be divided into hardware-based and software-based approaches. A straightforward hardware approach consists in increasing the workspace provided by each haptic device to fit that of the VE, either through a bigger frame or a redundant DoF. However the best result obtained for bimanual devices was the reach of human arms, in the cases of the DLR interface [3] and the Haptic Workstation [9]. Another solution for handling large VEs is the use of mobile haptic interfaces, i.e. haptic devices fixed on a mobile robot. Bimanual examples of these are the Mobile Haptic Grasper [12] and VISHARD7-based mobile interface [13]. However, while potentially providing an infinite planar workspace [11], these devices are still limited in vertical reach. Other hardware approaches solve the workspace issue by providing additional DoF to the user to handle navigation in the VE. For instance, a 3DoF foot pedal was used for controlling the motion of a two-armed robot in a remote environment [14].

While the hardware approaches do manage to solve the workspace issue to a certain extent, such devices are not necessarily widespread. Software approaches have the advantage of being generic and applicable to any haptic device available to the user with no further requirements, although the majority of them are not bimanual-specific. A first technique consists in applying a scaling factor to match the real workspace provided by the haptic devices with a virtual volume defined in the VE [15], although reducing the accuracy of motions in the virtual space. Another approach is the clutching technique, which consists in holding down a button to temporarily interrupt the coupling between the device and the proxy while the user recenters the device. The Dual Shell method is an extension of this technique, that automatically handles the clutching when predefined boundaries are reached, without requiring the potentially counterintuitive manipulation of a button [16]. The use of rate control was also proposed to control the velocity of the virtual proxy through the position of the haptic device [17]. This technique infinitely increases the workspace in all directions, however it is far from being intuitive and appears to be an acquired skill.

This leads to the Bubble technique, which uses position control inside predefined spherical boundaries of the device, and rate control when the device leaves those boundaries [18]. This technique was used more recently for bimanual interaction with complex VEs through the Haptic Workstation, by allowing users to translate and rotate the camera by moving both hands outside the bubble 
in the same direction [9]. This technique showed to be efficient for simultaneous navigation and manipulation with the Haptic Workstation, since it allows interaction with both palms and fingers. However, it remains more difficult to use with single-point interfaces. In this case, picked objects are frequently dropped during the translations of the virtual workspace through rate control, especially when using two different interfaces with physical workspaces of different size and shape.

The problem of grasping virtual objects with two single-point haptic interfaces has not yet been specifically addressed, as few bimanual techniques focus on two-handed haptic manipulation. In this context, several issues arise, although they have received little attention. Virtual springs between multiple contact points [6] were used for the haptic rendering of grasping, while repulsion forces $[3,8]$ were computed for the prevention of collisions between a user's hands and the haptic devices. Previous work from the area of augmented reality could be used for the modulation of the stiffness of objects [19] during a manipulation with haptic devices exhibiting different gains. Therefore, the question of how to facilitate the carrying of objects with dual single-point interfaces remains fully open, and we provide a first answer in this paper.

\section{Novel Techniques for Improving Bimanual Interaction with Dual Single-Point Haptic Interfaces}

We propose a set of new interaction techniques for improving the exploration of large VEs with two haptic interfaces, and the manipulation of objects with two 3 DoF haptic devices represented by simple proxies that enter in contact with virtual objects through single contact points. We first present two haptic exploration techniques: the double bubble, which allows free motion with both hands in a VE, and a viewport adaptation method that maintains both virtual proxies on screen at all times. Then, we present two haptic manipulation techniques: the magnetic pinch, which uses a simulated spring to keep the virtual proxies from dropping a picked object, and the joint control, which solves issues related to different control modes between the two hands.

\subsection{Double Bubble}

In the double bubble technique, the workspace of each haptic device is defined by two areas, each associated to a control mode. An inner area controls the proxy directly in position, and an outer area, starting at the boundaries of the inner area and extending up to the physical limits of the device, controls the virtual workspace in speed within the VE. Besides using two interfaces instead of one, two major differences separate the double bubble from the previously mentioned bubble technique. The first difference is the use of a rectangular parallelepiped for the boundaries of the bubbles instead of a sphere, to better fit the physical workspaces of the devices. We can notably think of PHANToM devices which have a higher width than their height or depth. The second difference is the 
presence of a visual feedback added to the haptic feedback when the devices leave the boundaries, in the form of a trail behind the rate-controlled proxies. The technique is illustrated in Figure 2.
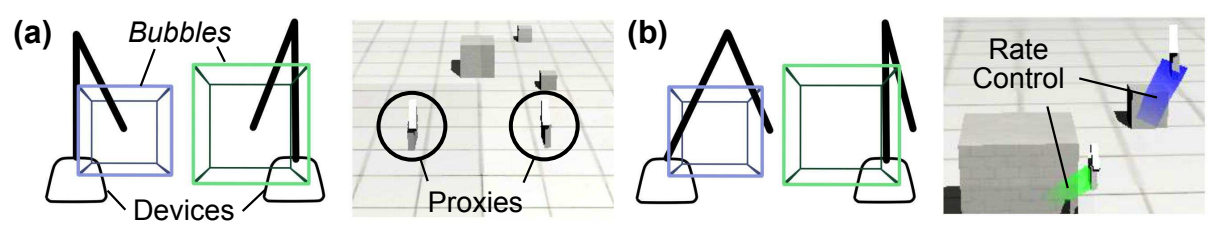

Fig. 2. Control modes of the double bubble. (a) Devices inside the bubbles : position control. (b) Devices outside the bubbles : rate control.

\subsection{Viewport Adaptation}

Since each device is attached to a bubble independent from the other, a method is required to keep both proxies on the screen, as these can move infinitely in completely opposite directions. Thus, we developed a method to ensure both virtual workspaces stay in the screen. This is accomplished by setting the distance of the camera to the center of the scene to a value proportional to the distance between the leftmost border of the left workspace and the rightmost border of the right workspace, plus an arbitrary margin (Figure 3). Given the left virtual workspace of center $\mathbf{l}=\left(l_{x}, l_{y}, l_{z}\right)$ and width $w_{l}$, and the right workspace of center $\mathbf{r}=\left(r_{x}, r_{y}, r_{z}\right)$ and width $w_{r}$, the position of the camera is computed following Equations (1-3).
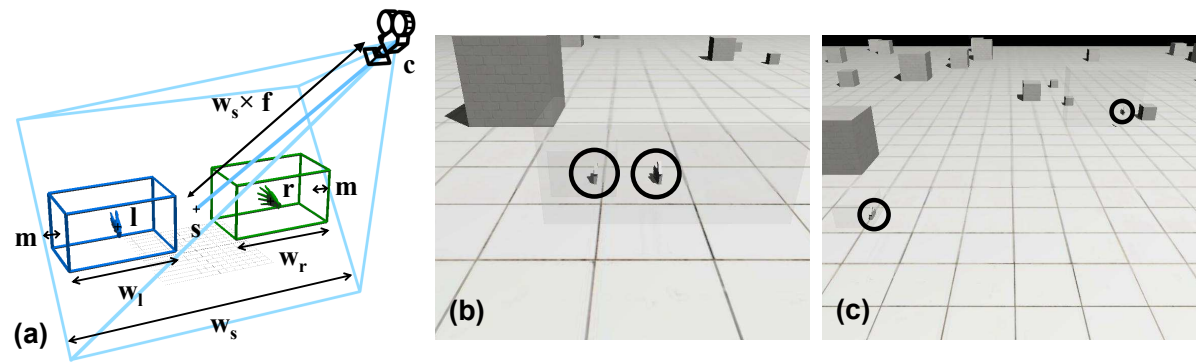

Fig. 3. Viewport adaptation. (a) Computation of the camera position. (b-c) Automatic viewport adaptation from different relative positioning of the proxies (circled).

The center of scene $\mathbf{s}$ is first computed from both workspace centers following:

$$
\mathbf{s}=\left(\frac{l_{x}+r_{x}}{2}, \frac{l_{y}+r_{y}}{2}, \max \left(l_{z}, r_{z}\right)\right) .
$$

The width of the displayed scene $w_{s}$ is then computed from the widths of both workspaces $w_{l}$ and $w_{r}$, as well as an arbitrary margin $m$ that ensures that the virtual workspace boundaries do not leave the borders of the screen: 


$$
w_{s}=\sqrt{\left(r_{x}-l_{x}\right)^{2}+\left(r_{y}-l_{y}\right)^{2}}+w_{l} / 2+w_{r} / 2+2 m .
$$

Finally, the position of the camera $\mathbf{c}$ is computed following:

$$
\mathbf{c}=\mathbf{s}+w_{s} \times d \times \mathbf{a} .
$$

where $d$ is a scalar that depends on the camera field of view, and $\mathbf{a}$ is an arbitrary vector that determines the angle from which the scene is displayed.

\subsection{Magnetic Pinch}

In order to facilitate the picking of virtual objects with two single-point interfaces, we propose two haptic manipulation techniques triggered whenever a grasping situation is detected. Three conditions are considered to determine whether both hands are grasping an object or not, according to the contact normals, the contact forces, and the relative position of both hands (Figure 4):

1. The angle between the contact normals must be under a certain threshold.

2. Both contact forces must exceed a threshold in order to discriminate simple contacts with an object from a true intent of grasping the object.

3. Two cylinders projected from both proxies following the contact normal and whose radii match the sizes of the proxies must intersect.
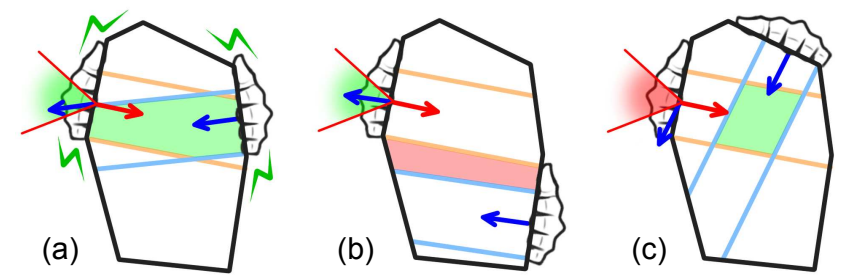

(d)

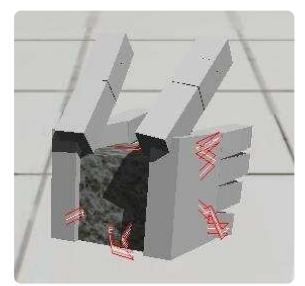

Fig. 4. Different cases of dual contact with a virtual object: (a) Normals nearly colinear and hands face-to-face, (b) Hands not in front of each other, (c) Normals far from colinearity. (d) Visual feedback of the magnetic pinch, symbolized by red bolts.

Once the grasping is initiated, the magnetic pinch takes effect, simulating a spring pulling both hands towards the picked object to prevent unintentional drops. For each haptic device, a force $\mathbf{F}_{h}$ is generated following:

$$
\mathbf{F}_{h}=-k_{h} \times\left(1-\frac{g_{s}}{\|\mathbf{o}-\mathbf{p}\|}\right) \times(\mathbf{o}-\mathbf{p}) .
$$

where $\mathbf{p}$ is the position of the first interface, $\mathbf{o}$ is the position of the second interface, $g_{s}$ is the size of the grasped object (the distance between the two contact points when the grasping is initiated), and $k_{h}$ is the stiffness of the spring. The spring is removed as soon as the user gives enough force to end the contact of the hands with the object, hence dropping it. 
Additionally, the position of the grasped object $\mathbf{g}_{p}$ can be constrained to the central point between the positions of the two virtual proxies $\mathbf{l}$ and $\mathbf{r}$, further reducing the risk of unwanted drops. For this, we use another spring of stiffness $k_{o}$, with a force $\mathbf{F}_{o}$, following:

$$
\mathbf{F}_{o}=-k_{o} \times\left(\frac{\mathbf{l}+\mathbf{r}}{2}-\mathbf{g}_{p}\right) .
$$

The spring feels as if the hands were "magnetized" to the object, and small red bolts are visually displayed to highlight this effect.

\subsection{Joint Control}

The double bubble metaphor may introduce a difference in control modes and/or scaling factors when activated. In order to reduce the impact of these differences when pick-and-placing a virtual object, we introduce the notion of joint control. During a grasping situation, both devices use a common control/display ratio (average of both) and common bubble size (minimal dimensions), and enter rate control simultaneously when at least one device leaves its bubble. This technique allows easier exploration of a VE when holding an object between virtual hands controlled by two different haptic interfaces.
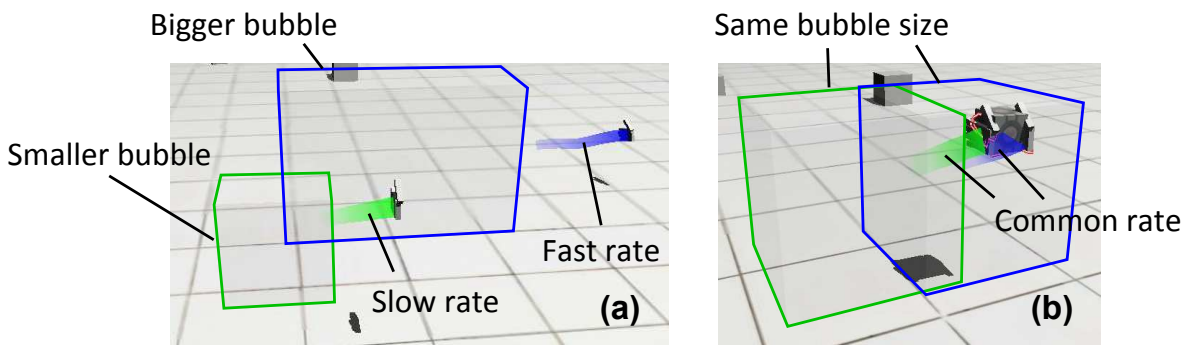

Fig. 5. Illustration of joint control. (a) Difference in bubble size and workspace translation speed without joint control. (b) Carrying an object with joint control.

\section{Evaluation}

To assess the efficiency of the proposed techniques, we conducted an experiment involving a simple pick-and-place task, where users had to pick a cube and place it at a given position. To evaluate the double bubble technique, we compared it to the clutching technique for workspace extension, and the benefits of the magnetic pinch and joint control were also measured for grasping facilitation.

\subsection{Method}

Population. Thirteen participants (2 females and 11 males) aged from 20 to 26 (mean $=22.8, \mathrm{sd}=1.7)$ performed the experiment. None of the participants 
had any known perception disorder. All participants were naïve with respect to the proposed techniques, as well as to the experimental setup and the purpose of the experiment.

Experimental Apparatus. The participants were seated at $1 \mathrm{~m}$ in front of a 24 inch widescreen monitor. The experiment was conducted using two different haptic interfaces. The participants manipulated a Falcon (Novint Technologies Inc., Albuquerque, New Mexico, USA) in their left hand, and a PHANToM Omni (Sensable Technologies, Wilmington, Massachusetts, USA) in their right hand, both placed in front of the screen as shown in Figure 6. Visual feedback was rendered at a refresh rate of $50 \mathrm{~Hz}$, while the haptic rendering rate was 1,000 Hz. Physical simulation was performed using Nvidia PhysX at a rate of 1,000 Hz to match the update frequency of the haptic loop. A virtual coupling mechanism was used between the haptic interfaces and the virtual proxies by simulating a spring-damper system between each haptic device and its corresponding proxy.

Virtual Environment. The VE was composed of a 100m-wide ground plane with four potential target planes, of $1 \mathrm{~m}$ of width, placed at the corners of a $6 \mathrm{~m}$-wide square around the center of the VE. The target plane of each trial were colored in red, and the other planes were colored in white. The cube to be manipulated had a width of $30 \mathrm{~cm}$ and a mass of $3 \mathrm{~g}$, and was placed at the center of the VE. The proxies controlled by each haptic device were physically represented by cubes of $20 \mathrm{~cm}$ of width, and were positioned $2 \mathrm{~m}$ away from each other and $5 \mathrm{~m}$ away from the central cube at the start of each trial. The cube was thus lying beyond the limits of the workspaces. The proxy controlled by the left device was visually represented by a blue left hand, and the right proxy was represented by a green right hand. Figure 6 shows the scene as displayed at the beginning of a trial.
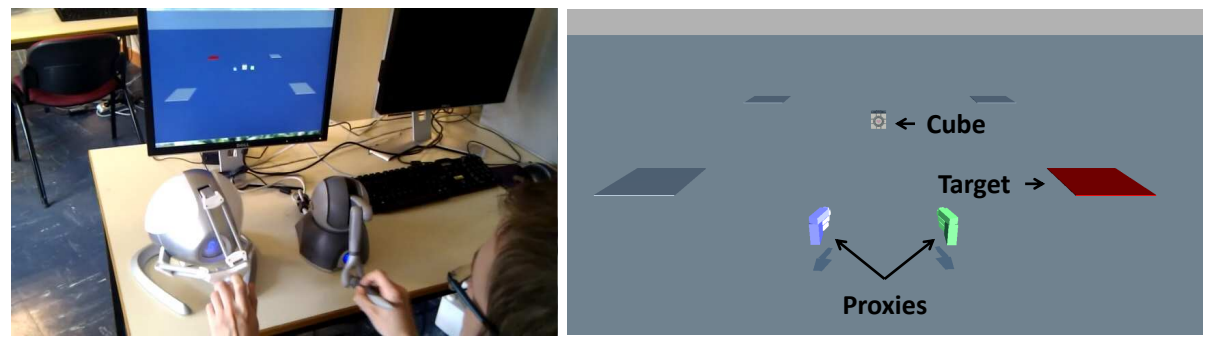

Fig. 6. Apparatus and virtual environment used in the experiment.

Procedure. At the start of each trial, both haptic devices and proxies were set to their starting positions. The subject had to pick the cube from both sides, carry it towards the red target and make the cube contact with the target, thus ending the trial. A black screen warned the subject about the beginning of the next trial. 
Experimental Conditions. We used a within-subject design to evaluate the four different conditions. In the control condition $\mathrm{Ctrl}$, the participants were able to use the clutching technique when they reached the limits of the workspaces. The three other conditions corresponded to: (1) DB (double bubble), (2) MP (clutching with magnetic pinch/joint control) and (3) DB+MP (a combination of double bubble and magnetic pinch/joint control). All the conditions were tested 44 times (11 times per target). The order between the different conditions was counterbalanced across participants, and for each condition, the order between the targets was randomized. The experiment lasted around 1 hour.

Collected Data. For each trial and each participant, the completion time and number of drops were recorded. The completion time is the time elapsed between the moment the proxies leave their starting positions and the moment the cube touches its target plane. The number of drops is the number of hits recorded between the cube and any part of the ground plane that is not the target plane. At the end of the experiment, participants had to complete a subjective questionnaire in which they had to grade the different techniques according to different criteria. The participants could rate the criteria from 1 (very bad) to 7 (very good). The different criteria were: (1) Global appreciation, (2) Efficiency, (3) Learning, (4) Usability, (5) Fatigue, and (6) Realism.

\subsection{Experiment Results}

Completion Time We conducted a statistical analysis from the completion time data collected during the experiment. For each participant, statistics (mean M, standard deviation SD) were computed on the 44 trials in each condition. A Friedman test on the completion time (in seconds) revealed a significant effect of the technique $\left(\chi^{2}=27.66, p<0.001\right)$. Follow-up post-hoc analysis revealed that completion time in both the $M P(M=14.16, S D=7.14)$ and $D B / M P$ $(M=8.43, S D=2.91)$ conditions were significantly shorter that in the control $(M=21.41, S D=13.19)$ and $D B(M=20.06, S D=14.63)$ conditions $(p<$ 0.001 in all cases), and that the $D B+M P$ condition led to significantly shorter times than the $M P$ condition as well $(p<0.001)$.

Number of drops Similarly, a statistical analysis was conducted on the number of drops for all trials of each participant. A Friedman test showed a significant effect of the technique $\left(\chi^{2}=25.52, p<0.001\right)$. Post-hoc analysis showed that the $M P(M=4.22, S D=9.45)$ and $D B / M P(M=2.36, S D=2.33)$ conditions led to significatively less drops than the control $(M=7.88, S D=6.37)$ and $D B$ $(M=8.79, S D=6.77)$ conditions $(p<0.001$ in all cases $)$.

\subsection{Subjective Questionnaire}

We perfomed a Friedman test to analyse the answers of the participants to the subjective questionnaire. The reported p-values were adjusted for multiple 
comparisons (alpha-level $\mathrm{p}=0.05)$. We found a significant effect for 5 criteria: Global appreciation $\left(\chi^{2}=4.62, p<0.001\right)$, Efficiency $\left(\chi^{2}=4.92, p<0.001\right)$, Learning easiness $\left(\chi^{2}=4.50, p<0.001\right)$, Use easiness $\left(\chi^{2}=4.80, p<0.001\right)$ and Fatigue $\left(\chi^{2}=4.46, p<0.001\right)$.

Post-hoc analysis showed that the $D B+M P$ condition was preferred to both the control and $D B$ for all criteria: Global appreciation $(p<0.001$ and $p<0.001$ respectively), Efficiency $(p<0.001$ and $p<0.001)$, Learning $(p<0.001$ and $p<0.001)$, Usability $(p<0.001$ and $p<0.001)$ and Fatigue $(p<0.001$ and $p<0.001)$. The $M P$ condition was also preferred over the control and $D B$ for 3 criteria: Global appreciation $(p=0.029$ and $p=0.028)$, Learning $(p=0.032$ and $p=0.009)$ and Usability $(p=0.027$ and $p=0.008)$, plus a fourth criteria for the $D B$ : Efficiency $(p=0.020)$.
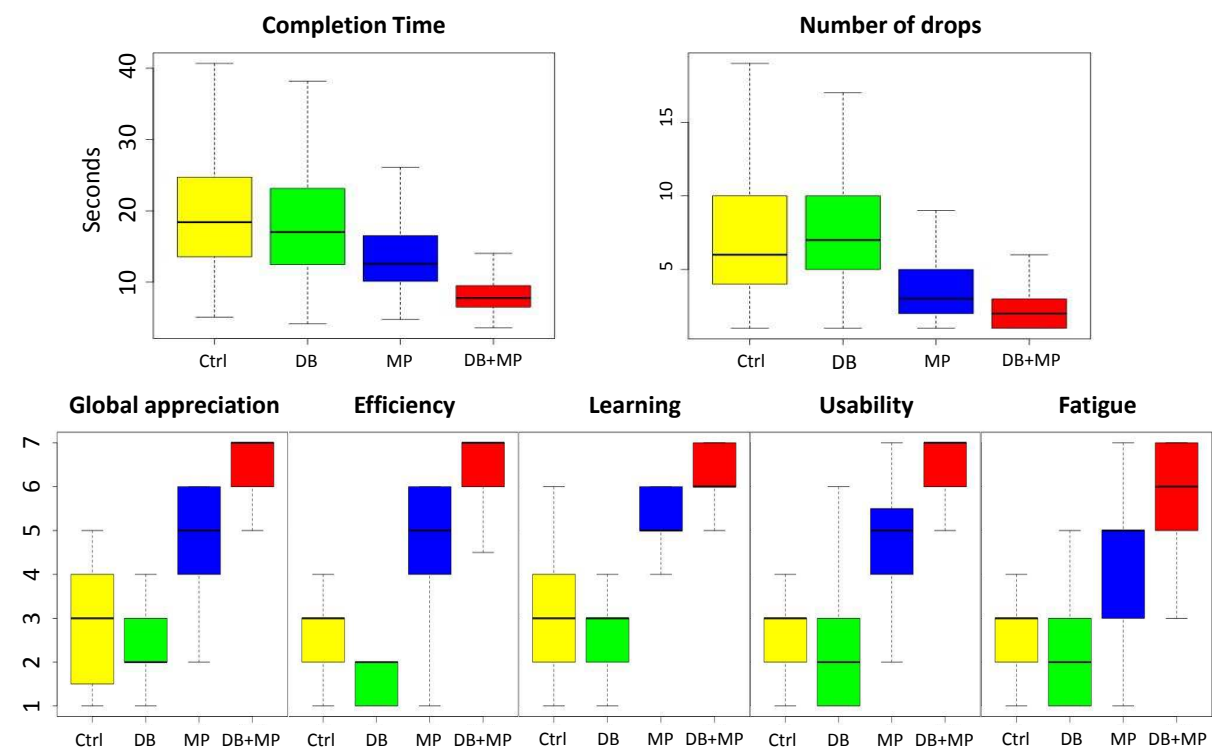

Fig. 7. Box plots of the completion times, number of drops and subjective ratings for the significative criteria, for all conditions. They are delimited by the quartile $(25 \%$ quantile and $75 \%$ quantile) of the distribution of the condition over the individuals. The median is represented for each trial.

\section{Discussion}

We proposed the double bubble technique with viewport adaptation for bimanual haptic navigation in a large VE as well as two haptic manipulation techniques, the magnetic pinch and joint control. The conducted experiment showed that the manipulation techniques improved performance and subjective appreciation for a pick-and-place task over the double bubble and clutching navigation techniques, while the combination of all of the proposed techniques led to the best results.

The double bubble, used alone, performed as good as the clutching technique without outperforming it, in terms of completion time, drop rate, and subjective 
appreciation. The technique allows to translate the workspace in a VE in a smoother way than the clutching technique, by removing the need to move the devices back and forth several times. We are planning to allow rotations of the viewport with the technique in future work.

The experiment showed that the magnetic pinch and joint control significantly reduced completion times and dropping rates compared to the conditions that did not use them. In addition, the subjective appreciation also favored the conditions which used these techniques over those that did not, globally and more particularly for learning and usability. These results strongly indicate that the magnetic pinch and joint control techniques, by stabilizing the grasping of a virtual object with virtual proxies, are efficient for facilitating pick-and-place tasks. Additionally, while the magnetic pinch inherently adds an unrealistic behaviour through the magnetic attraction, it does not seem to hinder the global realism of the scene, as no significant difference in the participants perception of realism was reported for the different conditions.

The best results were obtained with the combination of all of the proposed techniques. The double bubble showed its full potential when used jointly with the magnetic pinch and joint control, outperforming the combination of the latter techniques with clutching. The double bubble allows users to perform the task in a simpler and faster way than the clutching technique, which imposes frequent stops of both proxies to recenter the two haptic devices.

\section{Conclusion and Perspectives}

In this paper, we presented novel interaction techniques for bimanual haptic manipulation of virtual objects with two single-point haptic interfaces. The double bubble allows to move the workspaces of both virtual proxies inside the VE through hybrid position/rate controls, and a viewport adaptation technique keeps both proxies on screen at all times. The magnetic pinch prevents dropping of picked objects using a virtual spring between proxies. Finally, the joint control allows better handling of picked objects when moving around.

An experiment with a pick-and-place task showed that the magnetic pinch and joint control could lead to faster completion of the task with less unwanted drops of the object and overall better user appreciation compared to conditions that did not use them. They are thus efficient for simplifying the picking and carrying of an object. The double bubble, when used jointly with the aforementioned techniques, reduced even further the time needed to complete the task, outperforming the clutching technique. Overall, the combination of all of these techniques was shown to be very efficient for extending the workspaces of different haptic interfaces and allowing bimanual manipulation of objects with single-point interfaces in large VEs.

Future work will focus on supporting 6 DoF devices and more complex proxies that generate multiple contact points with objects. We also plan to increase the interaction possibilities of the techniques, and apply them to more complex tasks and applications like industrial prototyping or medical training. 


\section{References}

1. Sun L.-W., Van Meer F., Bailly Y., Yeung C. K.: Design and Development of a Da Vinci Surgical System Simulator. In: IEEE ICMA, pp. 1050-1055 (2007)

2. Li S., Frisoli A., Avizzano C., Ruffaldi E., Lugo-Villeda L., Bergamasco M.: Bimanual Haptic-desktop platform for upper-limb post-stroke rehabilitation: Practical trials. In: Proc. of IEEE ROBIO, pp. 480-485 (2009)

3. Hulin T., Sagardia M., Artigas J., Schaetzle S., Kremer P., Preusche C.: HumanScale Bimanual Haptic Interface. In: Proc. of the 5th Int. Conf. on Enactive Interfaces, pp. 28-33 (2008)

4. Faeth A., Oren M., Sheller J., Godinez S., Harding C.: Cutting, Deforming and Painting of 3D meshes in a Two Handed Viso-haptic VR System. In: IEEE VR, pp. 213-216 (2008)

5. Murayama J., Bouguila L., Luo Y., Akahane K., Hasegawa S., Hirsbrunner B., Sato M.: SPIDAR G\&G: A Two-Handed Haptic Interface for Bimanual VR Interaction. In: Proc. of EuroHaptics, pp. 138-146 (2004)

6. Garcia-Robledo P., Ortego J., Barrio J., Galiana I., Ferre M., Aracil R.: Multifinger Haptic Interface for Bimanual Manipulation of Virtual Objects. In: Proc. of IEEE HAVE, pp. 30-35 (2009)

7. Walairacht S., Koike Y., Sato M.: String-based Haptic Interface Device for Multifingers. In: Proc. of IEEE VR, p. 293. (2000)

8. Endo T., Yoshikawa T., Kawasaki H.: Collision Avoidance Control for a Multifingered Bimanual Haptic Interface. In: Proc. of Int. Conf. on Haptics, pp. 251-256. Springer-Verlag, Berlin Heidelberg (2010)

9. Ott R., De Perrot V., Thalmann D., Vexo F.: MHaptic: a Haptic Manipulation Library for Generic Virtual Environments. In: Proc. of Cyberworlds, pp. 338-345 (2007)

10. Barbagli F., Salisbry K. Jr., Devengenzo R.: Enabling multi-finger, multi-hand virtualized grasping. In: Proc. of IEEE ICRA, pp. 809-815 (2003)

11. Formaglio A., Prattichizzo D., Barbagli F., Giannitrapani A.: Dynamic Performance of Mobile Haptic Interfaces. IEEE Trans. on Robotics 24, 559-575 (2008)

12. de Pascale M., Formaglio A., Prattichizzo D.: A mobile platform for haptic grasping in large environments. Virtual Reality 10, 11-23 (2006)

13. Peer A., Buss M.: A New Admittance-Type Haptic Interface for Bimanual Manipulations. IEEE/ASME Transactions on Mechatronics 13, 416-428 (2008)

14. Peer A., Unterhinninghofen U., Buss M.: Tele-assembly in Wide Remote Environments. In: Proc. of IEEE/RSJ IROS (2006)

15. Fischer A., Vance J. M.: PHANToM haptic device implemented in a projection screen virtual environment. In: Proc. of Workshop on Virtual Environments, pp. 225-229. (2003)

16. Isshiki M., Sezaki T., Akahane K., Hashimoto N.: A Proposal of a Clutch Mechanism for 6DoF Haptic Devices. In: Proc. of ICAT, pp. 57-63. (2008)

17. Zhai S.: User performance in relation to 3D input device design. Computer Graphics 32, 50-54 (1998)

18. Dominjon L., Lecuyer A., Burkhardt J.M., Andrade-Barroso G., Richir S.: The "Bubble" Technique: Interacting with Large Virtual Environments Using Haptic Devices with Limited Workspace. In: Proc. of First Joint Eurohaptics Conference and Symposium on Haptic Interfaces for Virtual Environment and Teleoperator Systems, pp. 639-640. (2005)

19. Jeon S., Harders M.: Extending Haptic Augmented Reality: Modulating Stiffness during Two-Point Squeezing. Proc. of IEEE Haptics Symposium (2012) 